\title{
Urinary exosome-based androgen receptor-variant 7 detection in metastatic castration-resistant prostate cancer patients
}

\author{
Chao Wang ${ }^{1}$, Xiang Liu ${ }^{2}$, Hongyan $\mathrm{Li}^{2}$, Libo Zhao ${ }^{2}$, Guanyi Kong ${ }^{2}$, Jing Chen ${ }^{2}$, Zhi Li ${ }^{2}$, Jianfei Qi ${ }^{3}$, \\ Ye Tian ${ }^{4}$, Fengbo Zhang $^{4}{ }^{\wedge}$ \\ ${ }^{1}$ Department of Urology, Beijing Pinggu Hospital, Beijing Friendship Hospital, Capital Medical University, Beijing, China; ${ }^{2}$ Echo Biotech Co., \\ Ltd, Beijing, China; ${ }^{3}$ Department of Biochemistry and Molecular Biology, University of Maryland School of Medicine, Baltimore, MD, USA; \\ ${ }^{4}$ Department of Urology, Beijing Friendship Hospital, Capital Medical University, Beijing, China \\ Contributions: (I) Conception and design: F Zhang, G Kong; (II) Administrative support: Y Tian; (III) Provision of study materials or patients: X Liu, \\ H Li, L Zhao, J Chen, Z Li; (IV) Collection and assembly of data: C Wang, F Zhang; (V) Data analysis and interpretation: L Zhao, J Qi, G Kong; (VI) \\ Manuscript writing: All authors; (VII) Final approval of manuscript: All authors. \\ Correspondence to: Fengbo Zhang. Department of Urology, Beijing Friendship Hospital, Capital Medical University, No. 95 Yong'an Road, Xi’Cheng \\ District, Beijing 100050, China. Email: 13701319061@139.com.
}

Background: Androgen receptor variant 7 (AR-V7) detection provides important information for the clinical management of abiraterone in metastatic castration-resistant prostate cancer (mCRPC). We performed a non-invasive urine-derived exosomal AR-V7 analysis of mCRPC patients.

Methods: A total of $34 \mathrm{mCRPC}$ patients were recruited including 16 patients treated with abiraterone (ABI) with stable prostate-specific antigen (PSA)/radiograph response (the ABI-Sta group) and 18 were resistant to abiraterone (the ABI-Res group). Urine was collected from patients and healthy control patients for the analysis. Exosomal ribonucleic acid was isolated from urine. Urinary exosome-based androgen receptorvariant 7 was detected by quantitative real-time polymerase chain reaction assay. Characteristics of patients and survival data were collected. The correlation between AR-V7 expression and the therapeutic effect/ survival outcomes of abiraterone was analyzed.

Results: Urine is the ideal biological sample for exosome separation and AR full-length analysis. Positive urine-derived exosomal AR-V7 was detected in 32.4\% (11 of 34) of the mCRPC patients' urine samples. Positive AR-V7 was more common in the ABI-Res patients than the ABI-Sta patients (50.0\% vs. 12.5\%, respectively; $\mathrm{P}=0.009)$, and was associated with a higher PSA progression rate and poorer overall survival (OS) $(\mathrm{P}=0.0031$, and $\mathrm{P}=0.0012$, respectively).

Conclusions: The present study showed that the detection of urine-derived exosomal AR-V7 provides a sensitive and feasible clinical workflow. The predicting role of urine-derived exosomal AR-V7 in mCRPC patients should be further verified using studies with greater sample sizes.

Keywords: Androgen receptor (AR); splicing variant; castration-resistant prostate cancer (PCa); abiraterone; exosome

Submitted Nov 26, 2021. Accepted for publication Jan 30, 2022.

doi: $10.21037 /$ tau-21-1136

View this article at: https://dx.doi.org/10.21037/tau-21-1136

\footnotetext{
$\wedge$ ORCID: 0000-0003-2659-1710.
} 


\section{Introduction}

Prostate cancer $(\mathrm{PCa})$ is the second most common malignant cancer among men worldwide $(1,2)$. Almost all PCa patients develop resistance to androgen deprivation therapy (ADT), which leads to a lethal state known as metastatic castration-resistant PCa (mCRPC) (3). In many cases, the androgen receptor $(\mathrm{AR})$ axis remains active during the development of mCRPC, which suggests that the survival and growth of tumors still rely on the downstream signaling of the AR pathway (4). Abiraterone acetate blocks the intra-tumoral synthesis of androgens by selectively inhibiting

Cytochrome P450 17A1 (CYP171A) $(5,6)$ and has been shown to significantly improve the median overall survival (OS) of mCRPC patients in several clinical trials [e.g., COU-AA-301 (7), and COU-AA-302 (8)]. However, the clinical outcome of abiraterone has inter-individual variability.

Several pathological and molecular biomarkers have been used for the diagnosis, staging, and monitoring of mCRPC patients' responses to abiraterone. Among them, the expression of a constitutively active ligandindependent $\mathrm{AR}$ variant, such as $\mathrm{AR}$ variant 7 (AR-V7), has been conjectured to be the most common mechanism of abiraterone resistance. AR-V7 is derived from the premessenger ribonucleic acid (mRNA) of AR-full length (ARFL) through alternative splicing $(9,10)$. The result of ligand binding domain deletion is the transcription activation of AR downstream target genes and CRPC progression. AR-FL, distributing on most prostate cancer cells, could serve as an input in AR-V7 detection. Positive AR-V7 in $\mathrm{PCa}$ is strongly associated with resistance to AR signaling inhibitors and poor clinical prognosis (11) and its detection can be of strategic importance especially for mCRPC patients' resistance to AR signaling inhibitors. Thus, the detection of $\mathrm{AR}-\mathrm{V7}$ is valuable in prognosis evaluations and determining subsequent therapeutic agents (12).

Sensitive and specific bioanalytical methods are indispensable to the implementation of AR-V7 in clinical practice. AR and its splicing variants can be detected in both tumor tissue and body fluids. Tumor tissue can be acquired by biopsy, but this method is invasive, costly, and, most importantly, in many cases, the re-biopsy samples are unavailable or unanalyzable (13). Currently, circulating tumor cell (CTC)-based AR-V7 quantification is the most common liquid biopsy strategy $(14,15)$. However, CTC sample processing is time sensitive and requires highly specialized equipment. Additionally, this method is only available for CTC positive patients, and a considerable number of patients are excluded due to a low CTC burden or technical inaccessibility. Notably, both single-site biopsies and specific-antibody captured CTC sample processing do not fully considered the heterogeneity of PCa (16). As an alternative approach, AR-V7 RNA could also be extracted from whole blood for next bioanalysis, but this approach may be affected by the instability of free RNA in blood and the large amount of contaminating RNA $(17,18)$.

To date, exosome derived biomarkers have revolutionized the diagnosis of multiple pathologies, including cancer (19). Exosomes are small membranous vesicles with a diameter of 30-150 nm that are secreted into body fluid (including blood, cerebrospinal fluid, urine, and milk) by various organ cells. Extracellular vesicles (EVs) contain functional proteins and nucleic acids derived from the organs from which they originate (20). As exosomes show the molecular signature of their histogenesis, they have become a popular research subject $(21,22)$. A prospective study with a limited sample size found that plasma-derived exosomal RNA is a reliable source of AR-V7 that can predict resistance to hormonal therapy in $\mathrm{PCa}$ (23). Urine is the most ubiquitous body fluid that can be regenerated rapidly. Further, a direct physical connection between the prostate and ureter exists. Cho demonstrated the possibility and reliability of detecting AR-V7 in exosomes derived from urine with a small series of patients indicating positive AR-V7 expression is more common in mCRPC patients than that of mHSPC patients (24), although the survival data is undetailed.

A small number of mCRPC patients treated with abiraterone acetate were included in the present study, and the expression of urine-derived exosomal AR-V7 was detected. One of aims of this study is to examine the feasibility of urine-derived exosomal AR-V7 analysis workflow in mCRCPC patients. Another is to investigate the associations between survival outcomes and urinederived exosomal AR-V7 in mCRCPC patients. We present the following article in accordance with the MDAR reporting checklist (available at https://tau.amegroups.com/ article/view/10.21037/tau-21-1136/rc).

\section{Methods}

\section{Patients and healthy donor selection}

The study was officially approved by the ethics committee of the Beijing Friendship Hospital (approval ID: L-2018030). All procedures performed in this study involving human participants were in accordance with the Declaration 


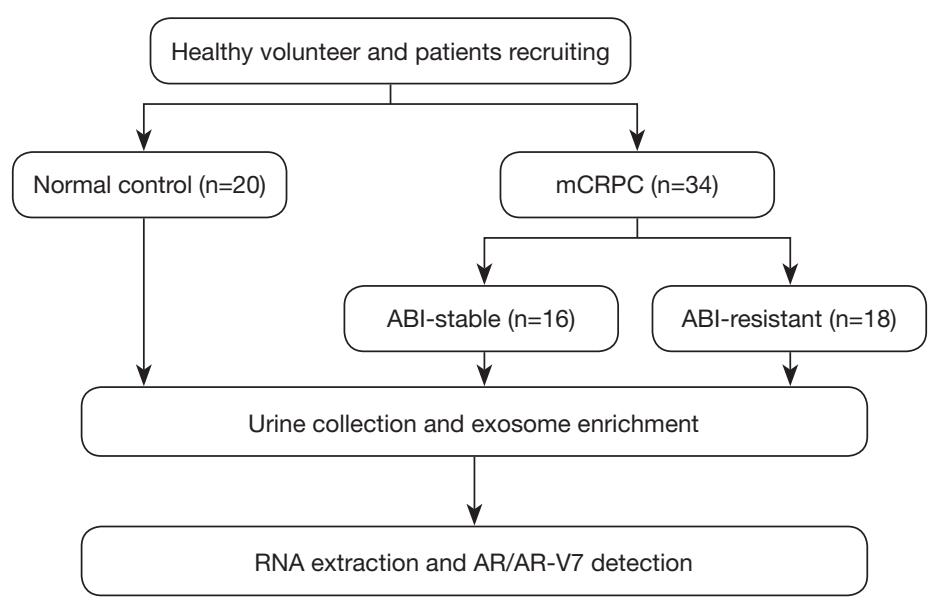

Figure 1 Framework of this project. mCRPC, metastatic castration resistant prostate cancer; ABI, abiraterone acetate; AR, androgen receptor; AR-V7, androgen receptor variant 7.

of Helsinki (as revised in 2013). Informed consent was taken from all the patients. The framework of this project is displayed in a schema graph (see Figure 1). A total of 34 metastatic CRPC patients treated with abiraterone acetate were included in this cross-sectional study. The all-inclusive regular-visit patients were histologically confirmed to have prostate adenocarcinoma, and castration levels of serum testosterone ( $\leq 20 \mathrm{ng} / \mathrm{dL}$ ) were maintained by ADT. Urine samples were collected for exosomal isolation after mCRPC was determined before the next-line treatment strategy. All the participants were categorized as being abiraterone stable (ABI-Sta) or abiraterone resistant (ABI-Res) as per the following definition of abiraterone resistance. Patients who had undergone docetaxel chemotherapy and radiotherapy previously were included in the study. All the patients were followed-up with, and the clinical outcomes were compared according to AR-V7 status.

In relation to disease progression, including prostatespecific antigen (PSA) and/or radiography progression according to the Prostate Cancer Working Group (PCWG) criteria, abiraterone resistance was defined as follows: (I) ongoing abiraterone treatment with at least 3 continuous rising serum PSA levels measured $\geq 1$ week apart and/ or radiography progression confirmed by a computed tomography $(\mathrm{CT}) /$ magnetic resonance imaging/bone scan consistent with the PCWG-2 guidelines; (II) ongoing abiraterone treatment $>3$ months and PSA flare should be excluded.

Patients' baseline characteristic and pretreatment outcome data, including age, Gleason score, previous treatment history, metastasis status, Eastern Cooperative Oncology Group (ECOG) performance status, serum PSA, alkaline phosphatase (ALP), lactate dehydrogenase (LDH), and hemoglobin, were collected. Serum PSA was measured in all patients at least once per month during treatment, and imaging evaluations were performed every 3 months or at the physicians' discretion.

A total of 20 healthy controls, including 9 males and 11 females, aged from 40-70 years old, were recruited for the study for whom no evidence of prostatic malignancy was found. An analysis of AR-V7 in primary/metastasis tumors was not performed, as this is not part of clinical practice.

\section{Urine collection and isolation of exosome}

For urine sample processing, $30 \mathrm{~mL}$ of morning urine was collected in an RNAse-free urine cup and stored at room temperature for $<4$ hours, and then centrifuged at 3,000 $\times \mathrm{g}$ for $30 \mathrm{~min}$. The supernatant was then transferred to a new $50 \mathrm{~mL}$ centrifuge tube and kept at $-80^{\circ} \mathrm{C}$.

The exosomes were isolated from the urine sample following a modified protocol as previously reported (19). Briefly, $30 \mathrm{~mL}$ of fresh urine was centrifuged at $3,000 \times \mathrm{g}$ for $30 \mathrm{~min}$ to eliminate the cell debris and insoluble particles. Polyethylene glycol (PEG)-6000 (with concentration of $40 \%$ ) was added to the urine at a final concentration of $8 \%$, which was then homogeneously mixed and left overnight. Next, the PEG-urine complex was centrifuged at 5,000 $\times \mathrm{g}$ for $20 \mathrm{~min}$, and the exosomal pellets were re-suspended in $100 \mu \mathrm{L}$ of $1 \times \mathrm{PBS}$, and stored at $-80^{\circ} \mathrm{C}$ for further analysis. 


\section{NTA and TEM}

Vesicle suspensions with concentrations between $1 \times 10^{7} / \mathrm{mL}$ and $1 \times 10^{9} / \mathrm{mL}$ were examined using the ZetaView PMX 110 (Particle Metrix, Meerbusch, Germany) equipped with a $405-\mathrm{nm}$ laser to determine the size and quantity of the isolated particles. A 60 -sec video was taken with a frame rate of 30 frames/sec, and particle movement was analyzed using nanoparticle tracking analysis (NTA) software (ZetaView 8.02.28). A total of $20 \mu \mathrm{L}$ exosome solution was placed on a copper mesh and incubated at room temperature for $10 \mathrm{~min}$. After washing with sterile distilled water, the exosome solution was contrasted with uranyl-oxalate solution for $1 \mathrm{~min}$. The sample was then dried for $2 \mathrm{~min}$ under incandescent light. The copper mesh was observed and photographed under a transmission electron microscope (TEM) (JEOL-JEM1400, Tokyo, Japan).

\section{Immunoblotting and exosome confirmation}

The exosomes were lysed by radioimmunoprecipitation assay lysis buffer, $30 \mu \mathrm{g}$ of total protein was boiled in $2 \times$ sodium dodecyl sulphate-polyacrylamide gel electrophoresis (SDS-PAGE) loading buffer for $5 \mathrm{~min}$ and used for immunoblotting. Protein samples were separated by 4-20\% SDS-PAGE and transferred to polyvinylidene-fluoride membranes using the tank method. The primary antibodies used in this experiment were as follows: anti-CD63 (Santa Cruz Biotechnology, sc-59284), anti-Alix (Abcam, ab117600), and anti-TSG101 (Abcam, ab125011). CD63 and Tsg 101 were used to positively confirm exosomes derived from urine. Calnexin was used as the negative control.

\section{Urine-derived exosomal RNA isolation and quantitative RT-PCR}

The total RNA of exosome was extracted using QIAzol Lysis Reagent (QIAGEN, 79306) according to the manufacturer's protocol. Total RNA was converted to complementary deoxyribonucleic acid (cDNA) using Superscript II reverse transcriptase (Invitrogen Life Technologies, 18064-014) in accordance with the manufacturer's instructions. The AR-FL was used as an internal control. The abundance of AR-FL and AR-V7 were detected by TaqMan real-time polymerase chain reaction (RT-PCR), and the sequences of primers and probes were as follows: AR-FL, forward primer 5'-CATCAAGGAACTCGATCGT-3', the forward primer 5'-GAACTGATGCAGCTCTCTC-3', probe
5'-ACATCCTGCTCAAGACGCTCCT-3'. AR-V7, forward primer 5'-CTGTGCGCCAGCAGAAAT-3', the forward primer 5'-TCAGGGTCTGGTCATTTTGA-3', probe 5'-TGTCCATCTTGTCGTCTTCG-3' (23). Each RT-PCR sample was reacted twice to confirm the results.

\section{Standard curve preparation}

Briefly, the cDNA of AR-FL or AR-V7 was inserted into vector $\mathrm{pBM}-8 \mathrm{~A}$. The insert was cut from the plasmid and in vitro transcription was performed. After the RNA was extracted using QIAzol lysis reagent and a concentration quantification by nanodrop, the RNA was diluted in $\log$ increments from $1 \times 10^{6}$ to 10 copies $/ \mu \mathrm{L}$. Next, TaqMan fluorescent quantitative PCR was conducted with different RNA concentrations, and the cycle threshold values (CT values) were collected.

\section{Statistical analysis}

All the statistical analyses were conducted using SPSS software (SPSS Inc., Chicago, IL, USA). All error bars represent mean \pm standard deviation $(\mathrm{SD})$, and were derived from 3 independent experiments. The continuous data are presented as mean $\pm \mathrm{SD}$, and independent samples $t$-tests were used for comparisons. The categorical data are presented as frequency and percentage, and were evaluated the using chi-square tests. Time-to-event outcomes [PSA/radiographic progression-free survival (PFS) and OS] were analysed using the Kaplan-Meier method, and survival-time differences were compared by log-rank tests. In all cases, $\mathrm{P}$ values $<0.05$ were considered statistically significant.

\section{Results}

\section{Urine-derived exosomal AR-FL and AR-V7 RNA expression in healthy volunteers}

To determine whether urine could be used for exosome analysis, we first performed exosome isolation by PEGurine precipitation $(30 \mathrm{~mL}$ for each test). The results showed that urine was an ideal biological sample for exosome enrichment. First, the western blot analysis of the common exosome markers indicated that exosome protein markers CD63 and TSG101 in the urine-derived exosomes were significantly enriched (see Figure $2 A$ ). Second, the morphological analysis by TEM showed a classical double membrane structure in exosomes collected from urine with 
A

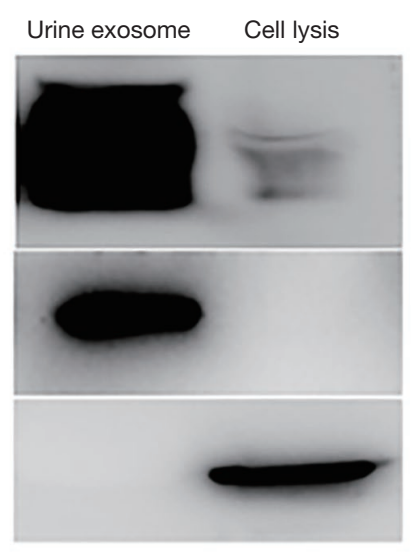

CD63

Tsg101

Calnexin

C

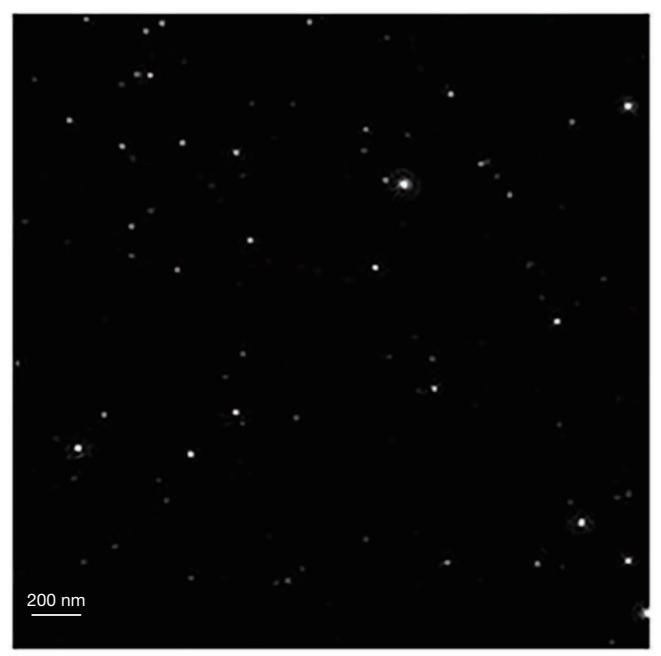

B

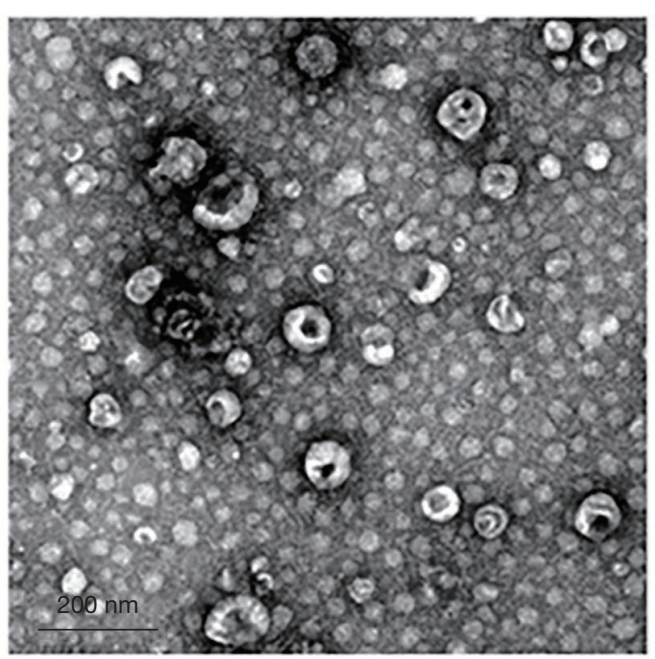

D

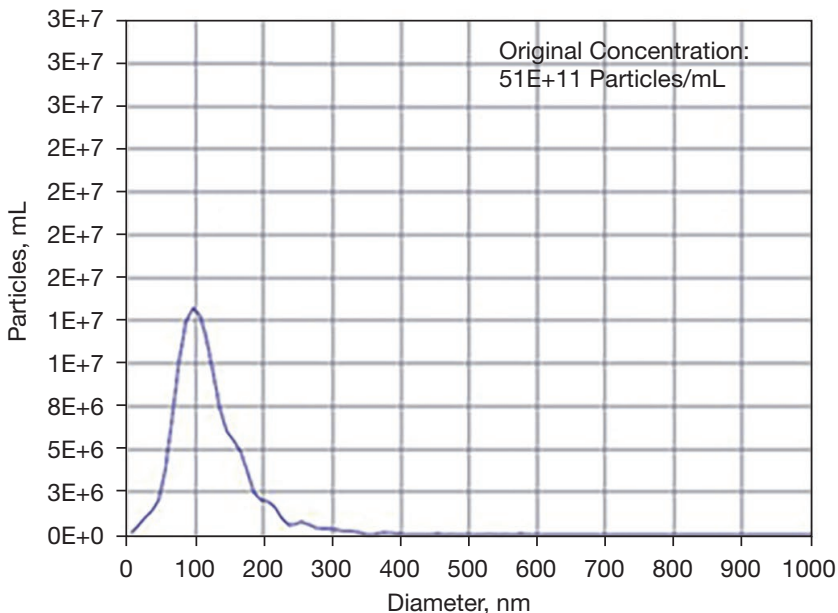

Figure 2 Exosome extraction from urine. (A) Immunoblotting of exosome marker CD63 and Tsg101 in exosomes derived from urine. Calnexin, which is a protein-located cytoplasm that is not present in exosomes, was used as the negative control; (B) representative TEM image of exosomes derived from urine; (C) representative NTA image of exosomes derived from urine; (D) size distribution and particle concentration. TEM, transmission electron microscope; NTA, nanoparticle tracking analysis.

an average diameter of $88.9 \pm 9.81 \mathrm{~nm}$ (see Figure $2 B$ ). Third, the NTA showed a concentration of exosomes around $5 \times 10^{10}$ particles $/ 30 \mathrm{~mL}$ urine, which was an acceptable abundance for further protein and RNA analysis (see Figure 2C,2D).

This study also investigated AR-V7 in the urine exosomes of mCRPC patients. First, we constructed a standard curve reflecting the copy number of AR-V7 and AR-FL in each PCR tube, and tested the detection limit of our system. According to our standard curve, we could detect as few as 10 AR-FL copies and AR-V7 plasmid DNA in each reaction (see Figure 3A,3B). Next, we enrolled 20 healthy controls. Total exosomal RNA was extracted for reverse transcription and further quantitative RT-PCR. We successfully detected AR-FL in each of the 20 urine exosome samples of the healthy volunteers (mean CT value: 30.85 ). The cutoff value for $\mathrm{AR}-\mathrm{V} 7$ was set as 36 to avoid false positive (see Figure $3 C$ ). These results were logical, as AR-FL is mainly expressed in a hormone deprivation state, but not in normal physiological conditions. These results proved that urinederived exosome enriched prostate-associated genetic material might be suitable for AR-V7 detection. 
A

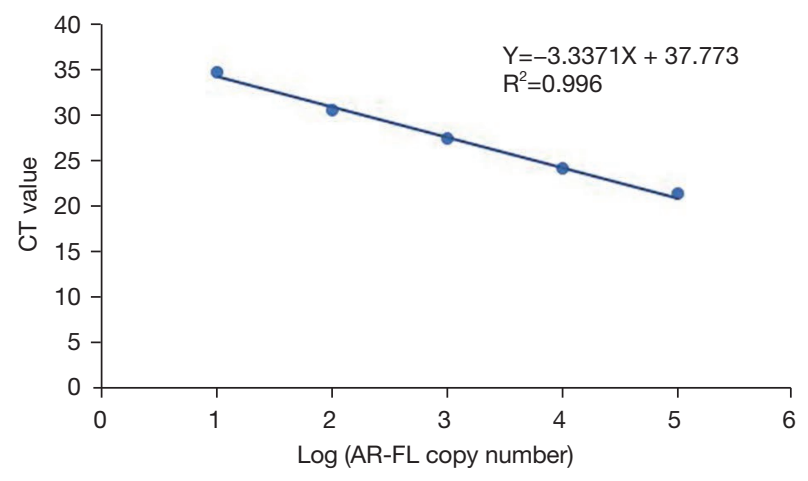

B

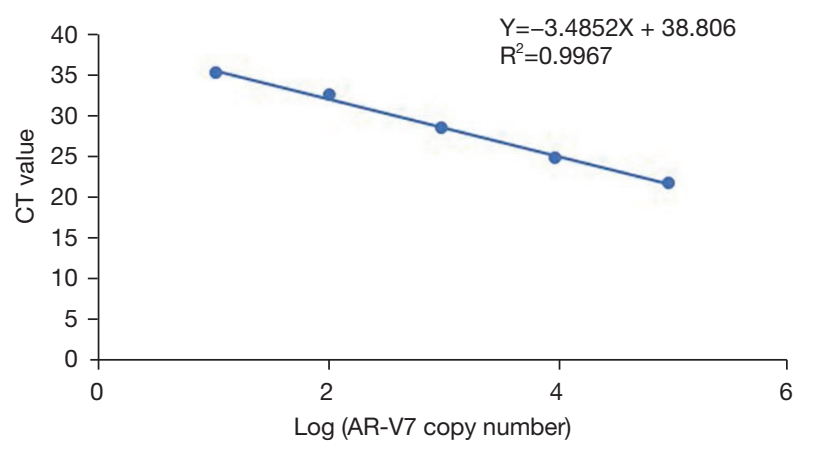

C

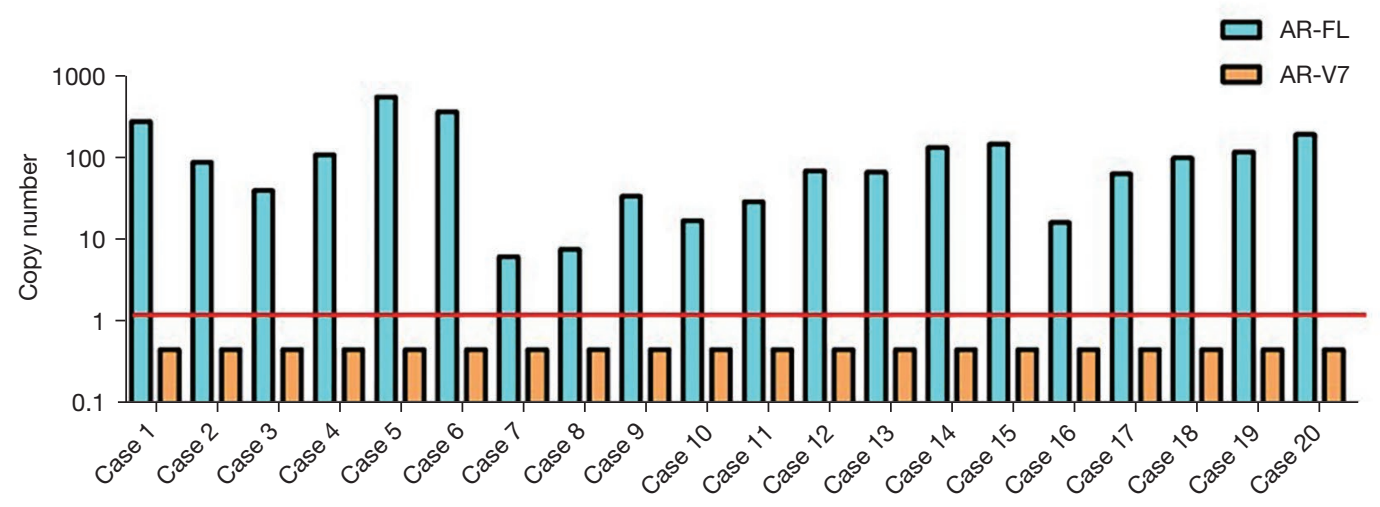

Figure 3 AR-FL/AR-V7 detection in urine of healthy volunteers. (A,B) Standard curve of AR-FL (A), AR-V7 (B) copy number and corresponding CT value in the RT-PCR quantification experiment. (C) Quantification of the expression level of AR-FL and AR-V7 in exosomes derived from the urine of healthy volunteers; the data are displayed as copy numbers $/ 14 \mathrm{~mL}$ urine. Negative samples were assigned a CT value of 40; the cutoff for AR-V7 positive results is shown by the red line. AR, androgen receptor; AR-V7, androgen receptor variant 7; AR-FL, full length androgen receptor; RT-PCR, real-time polymerase chain reaction; CT value, cycle threshold value

\section{Clinical characteristics and the expression of $A R-V 7$ in urine-derived exosomal RNA}

Overall, all of the 34 samples were positive for AR-FL, with an average CT value of 31.55 (74 copies/14 mL), and 11 samples tested positive for the expression of AR-V7 with a CT value of 33.02-37.42 (2.5-45 copies/14 mL). The baseline characteristics for the participants are reported in Table 1. Sixteen patients were categorized as abiraterone stable (the ABI-Sta), and 18 patients were categorized as abiraterone resistant (ABI-Res) as per the inclusion criteria. The median follow-up time was 15.7 months (range, 3.021.0 months). Our data identified the percentage of PSA progression, radiography progression and both are $55.56 \%$ (10/18), $22.22 \%(4 / 18)$ and $22.22 \%(4 / 18)$ of 18 ABI-Res patients vs. $25.0 \%$ (4/16), $12.5 \%(2 / 16)$ and $62.5 \%(10 / 16)$ of $16 \mathrm{ABI}-\mathrm{Sta}$ patients, respectively. Compared to the ABI-
Sta group, patients in the ABI-Res group had a higher proportion of prior docetaxel-based chemotherapy (33.33\% vs. 6.25\%), a higher median initial PSA level (241.24 vs. $84.22 \mathrm{ng} / \mathrm{mL}$ ), a higher median serum ALP level (420.71 vs. 205.51 U/L), and a higher median LDH level (143.31 vs. $125.62 \mathrm{U} / \mathrm{L})$. Positive AR-V7 was more common in the ABI-Res group than the ABI-Sta group $(50.0 \%$ in the ABI-Res group vs. $12.5 \%$ in the ABI-Sta group; $\mathrm{P}=0.009$; see Figure $4 A, 4 B)$. In addition, the presence of visceral metastasis was common in the ABI-Res group (7/18, $38.89 \%$ ), especially in the ABI-Res AR-V7(+) subgroup (5/9, $55.56 \%)$.

To further analyze the results, both groups were allocated to AR-V7(+) and AR-V7(-) subgroups according to the results of the AR-V7 analysis. The subgroup data showed that patients in the ABI-Res AR-V7(+) subgroup had a more invasive disease with higher initial PSA levels at diagnosis 


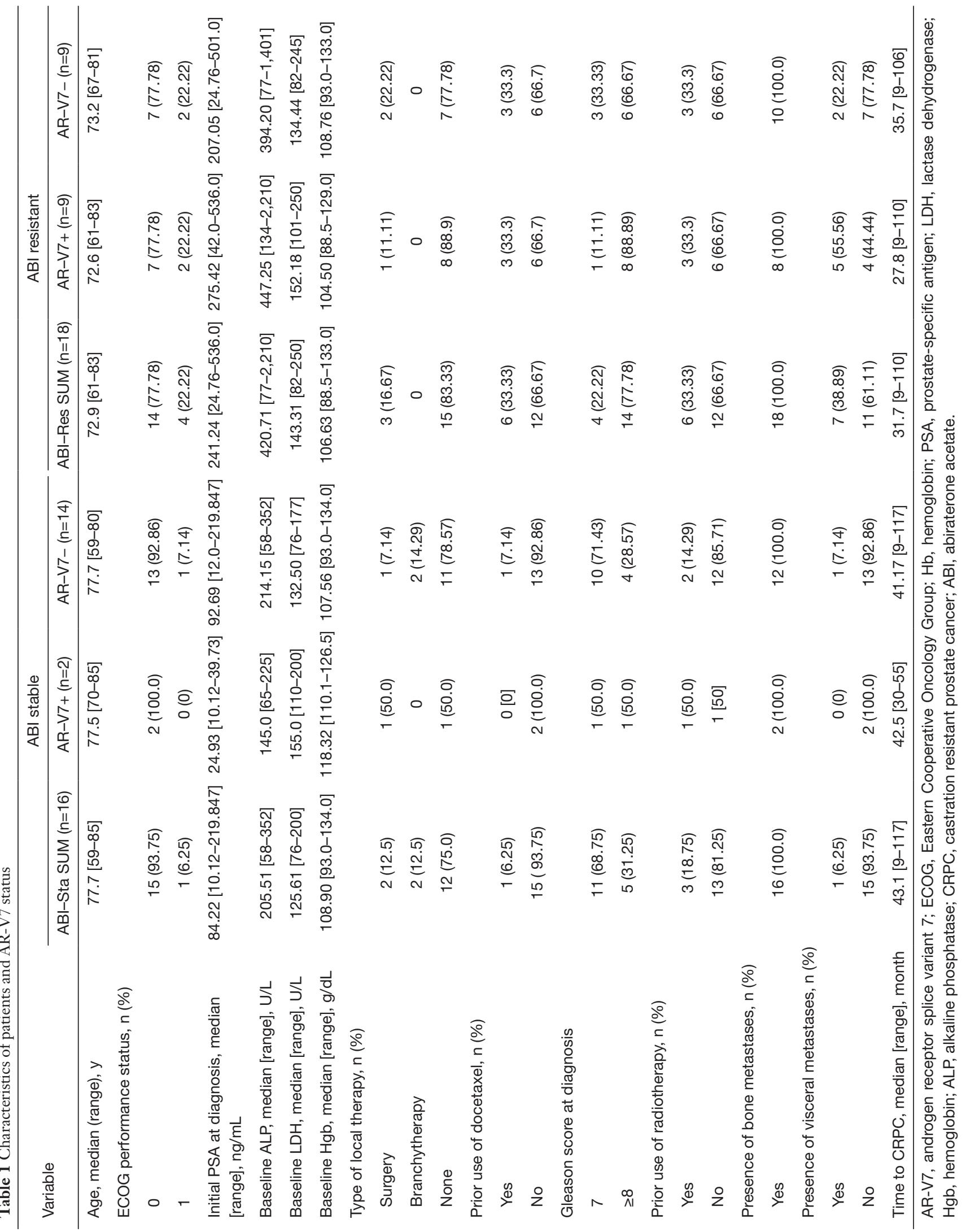



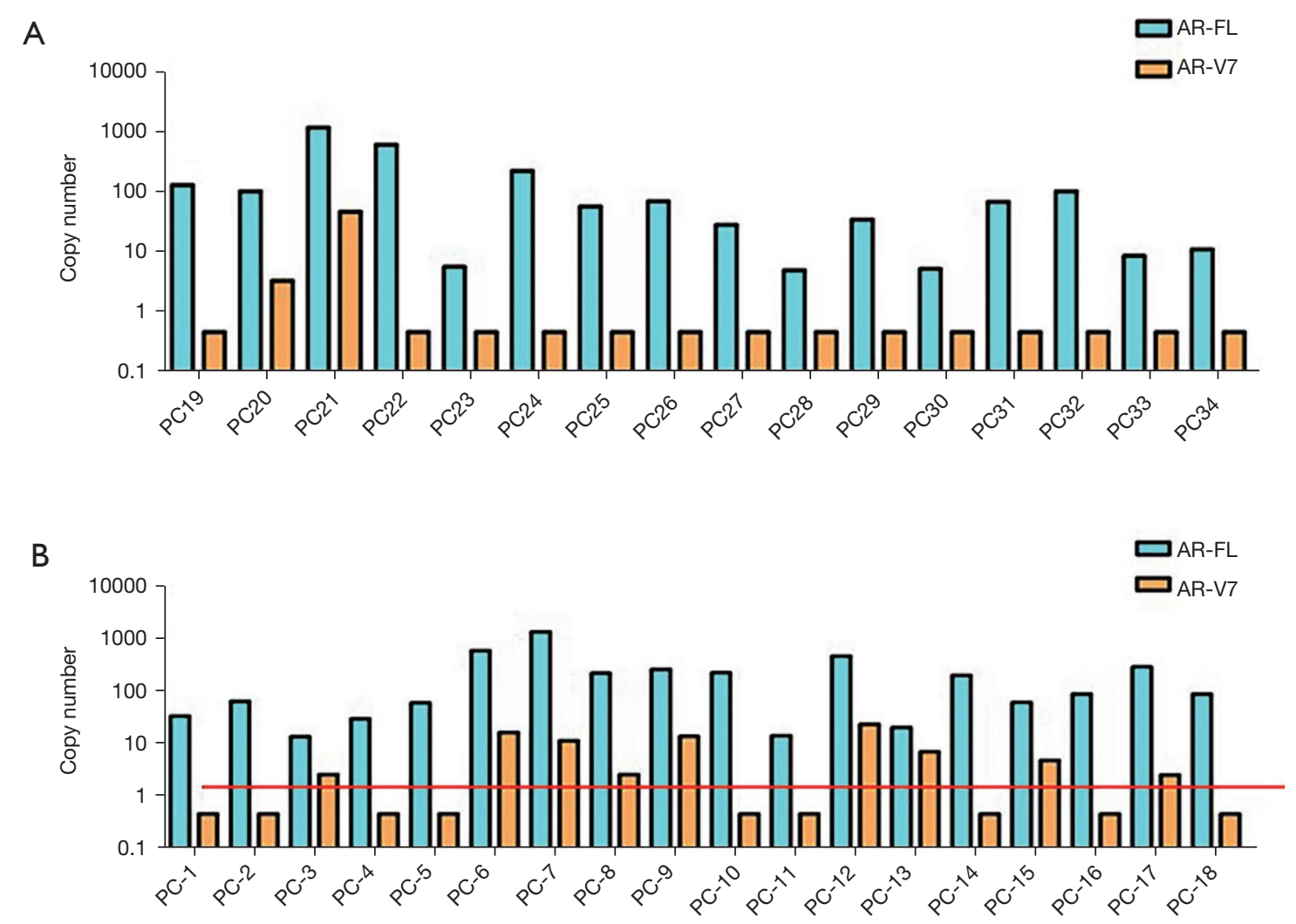

Figure 4 AR-FL/AR-V7 detection in urine-derived exosomes of Pca. (A,B) Quantification of the expression level of AR-FL and AR-V7 in exosomes derived from the urine of the ABI-Sta group and the ABI-Res group; the data are displayed as copy numbers/14 mL urine. Negative samples were assigned a Ct value of 40; the cutoff for AR-V7 positive results is shown by the red line. AR, androgen receptor; ARV7, androgen receptor variant 7; AR-FL, full length androgen receptor; ABI, abiraterone acetate.

(275.42 vs. $207.05 \mathrm{ng} / \mathrm{dL})$ and a shorter ADT time to CRPC (27.75 vs. 35.67 months) than those in the ABI-Res AR-V7(-) subgroup.

The Kaplan-Meier analysis did not confirm the role of AR-V7 as an independent prognostic factor for OS [hazards ratio (HR): 1.645; 95\% confidence interval (CI): 0.611-4.428; $\mathrm{P}=0.3089$ ] and PSA PFS (HR: $1.821,95 \%$ CI, 0.797-4.162; $\mathrm{P}=0.1133$ ), but this is probably due to the small number of patients enrolled in the study (see Figure 5). However, an analysis at the 20-month timepoint revealed that $10 / 11(90.91 \%) \mathrm{AR}-\mathrm{V} 7(+)$ patients experienced an increase $\geq 50 \%$ in PSA response $(\mathrm{P}=0.0031)$ and/or radiography progression vs. 14/23 (60.87\%) AR-V7(-) patients $(\mathrm{P}=0.0012)$.

\section{Discussion}

AR-V7 was coupled with AR gene transcription and facilitated by AR gene amplification/rearrangement. The positive expression of AR-V7 was found to be associated with resistance to the therapeutic target of the AR pathway (abiraterone and enzalutamide) in CRPC patients. AR$\mathrm{V} 7$ and the prognosis of $\mathrm{mCRPC}$ patients was positively correlated. Unfortunately, re-biopsies to obtain PCa tissue are often unacceptable in clinical practice. Most clinicians prefer to conduct liquid biopsies for detection, as they have a number of advantages, including that they are noninvasive, the require biomaterial is available, and can represent tumor heterogeneity. Blood sample-derived RNA from both CTCs and exosomes has been used to interrogate tumor biology. The clinical value of AR-V7 detection in urine remains unclear, but was tested in an individual study with a small number of samples (24).

We hypothesized that AR-V7 detection in EVs could be an alternative option to CTCs for evaluating the prognosis of mCRPC patients. The feasibility of isolating EVs from 
A

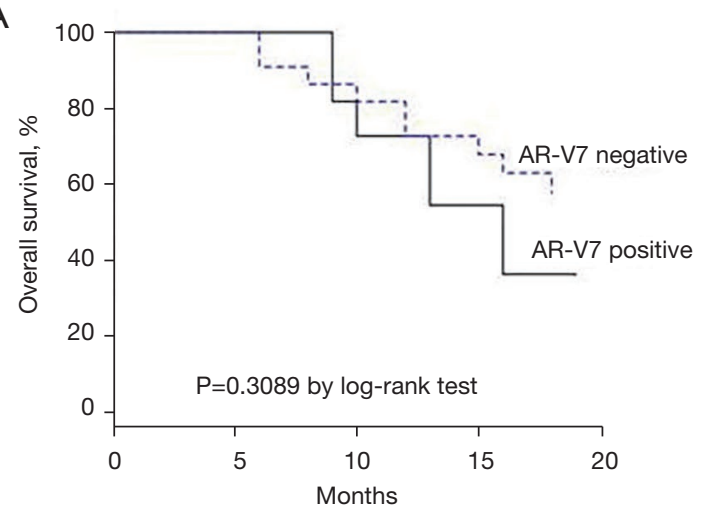

B

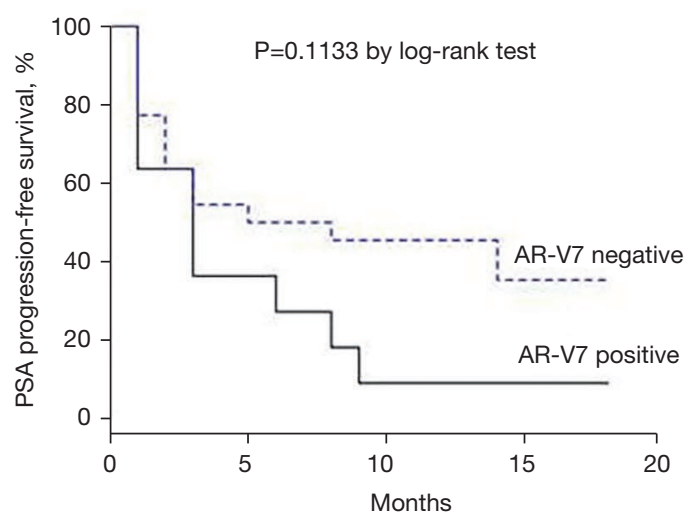

Figure 5 OS and PSA PFS curves according to the AR-V7 analysis. (A) OS curve; (B) PSA PFS curve; the difference between AR-V7 positive and negative groups was not significant, but this is probably due to the small number of patients enrolled in the study $(\mathrm{P}=0.3089$ and $\mathrm{P}=0.1133$, respectively). However, a significant difference was observed in the PSA PFS rate and OS rate at the follow-up endpoint ( $\mathrm{P}=0.0031$, and $\mathrm{P}=0.0012$, respectively, by a chi-square test). OS, overall survival; PSA, prostatic specific androgen; PFS, progression-free survival; AR, androgen receptor; AR-V7, androgen receptor variant 7.

urine was confirmed in the present study. We described our workflow for urine exosome extraction and exosomal AR-V7/AR-FL detection for which we used quantitative RT-PCR instead of droplet digital PCR. Healthy controls were enrolled to define the cutoff values for AR-V7 and AR-FL. Next, we analyzed mCRPC patients treated with abiraterone to explore the correlation between urine exosomal-derived AR-V7 and the therapeutic effect of abiraterone in mCRPC. Our results revealed that AR-V7 in urine-derived exosomes is closely associated with mCRPC clinical prognosis. Positive AR-V7 was more common in patients in the ABI-Res than the ABI-Sta group. Moreover, positive AR-V7 appears to be related to higher initial PSA levels at diagnosis, higher Gleason scores, and shorter ADT time to CRPC and survival.

The expression of AR-V7 in plasma-derived exosomes in $36 \mathrm{mCRPC}$ patients, and detected positive AR-V7 in $39 \%$ of the patients and found that it was associated with a poor clinical prognosis (23). The RNA recovered from plasma-derived exosomes could benefit the CRPC diagnostic workflow and minimize the negative effect of tumor heterogeneity. Given that the efficient isolation of tumor-specific materials from whole blood remains a major challenge due to the interruption of abundance contaminants, such as cells, vesicles, and proteins from healthy tissues, a more sensitive and reliable type of liquid biopsy for AR-V7 detection is needed. Woo et al. demonstrated their workflow for detecting AR-V7 mRNA expression in urinary EVs in PCa patients using ddPCR assays. No clinic follow-up data were available, but a greater tendency for higher AR-V7 expression in urine-derived EVs from patients with CRPC than in those with hormone sensitive $\mathrm{PCa}$ (24).

AR-V7 detection in plasma-derived exosomes was not performed due to the primary aim of this study is to develop a modified method for AR-V7 detection using urinederived exosome. Further study to evaluate the specificity and sensitivity of AR-V7 detection is needed to compare to that of plasma-derived exosomes. The present study conducted AR-V7 RNA detection with isolated urinederived exosomes using quantitative RT-PCR assays. The $50 \%$ positive rate of $\mathrm{AR}-\mathrm{V} 7$ in the urine-derived exosomes of ABI-Res patients demonstrates the detection sensitivity of the technology. Theoretically, plasma samples contain a large amount of non-cancer related exosomes in the plasma secreted by leucocytes, platelets, and red blood cells, which might lead to high frequency false positive detections of AR-V7. However, most of the biological macromolecules from the blood can be excluded by the renal corpuscle, and the urine contain more biomolecules. In this respect, urine-derived exosomes showed certain advantages over blood-derived exosomes in the monitoring of urinary tractrelated cancers, such as PCa. Initially, we conjectured that most EVs were secreted from shedding cells of the urethra epithelium, which may contain more PCa cell EVs. However, after examining 2 positive AR-V7 patients with previous radical proctectomy, we found that exosomes may be secreted by the renal corpuscle or urothelial cells.

This study had a number of limitations. First, it was not 
a prospective randomized research study, and only a small number of patients were included. Further, our KaplanMeier analysis did not reveal significant results in relation to OS and PFS; however, this is probably due to the small number of patients included in the study. An analysis of PSA progression and/or radiography progression rates at the 20-month timepoint showed that positive AR-V7 appeared to be associated with higher PSA progression and/ or radiography progression rates $(90.91 \%$ vs. $60.87 \%)$. A large-scale prospective trial now needs to be conducted to validate the findings of this pilot study.

The detection of AR-V7 has not been routinely applied in clinical practice until now probably due to a missing golden standard. The present study found AR-V7 detection in urine-derived exosomes could be a sensitive and convenient assay. Despite the elevated $\mathrm{AR}-\mathrm{V} 7$ positive proportion in this series, $50.0 \%$ of the ABI-Res CRPC patients did not have AR-V7 expression. These results remind us of the diversity of the molecular mechanism underlying abiraterone tolerance. Other alternatively spliced transcripts of ARs, such as ARV3 and ARV9, could also be involved in the development of abiraterone resistance. According to our results, urine-derived exosomes contain abundant molecular information relevant to prostatic cancer tissues, which could be used as biomarkers for screening related to abiraterone resistance. Finally, due to the unreachability of enzalutamide, the present study mainly focused on abiraterone resistance, and thus a similar conclusion cannot be drawn in relation to patients treated with enzalutamide.

\section{Conclusions}

The present study showed that the detection of urinederived exosomal AR-V7 is a non-invasive clinical workflow that is sensitive and feasible. The role of urine-derived exosomal AR-V7 in mCRPC patients should be further verified using studies with greater sample sizes.

\section{Acknowledgments}

Funding: Dr. Fengbo Zhang received financial support from the Beijing Natural Science Foundation (No. 7192044), which was used to maintain the exosome isolation, conduct the AR-V7 analysis, and review the manuscript. Dr. Chao Wang received funding from the Beijing Pinggu Hospital Research Foundation (No. pgyyqn2019-03), which was used to support his role in the study's data analysis and patient management.

\section{Footnote}

Reporting Checklist: The authors have completed the MDAR reporting checklist. Available at https://tau.amegroups.com/ article/view/10.21037/tau-21-1136/rc

Data Sharing Statement: Available at https://tau.amegroups. com/article/view/10.21037/tau-21-1136/dss

Conflicts of Interest: All authors have completed the ICMJE uniform disclosure form (available at https://tau.amegroups. com/article/view/10.21037/tau-21-1136/coif). CW reports that the manuscript was supported by Beijing Pinggu Hospital Research Foundation (No. pgyyqn2019-03). FZ reports that the manuscript was supported by the Beijing Natural Science Foundation (No. 7192044). XL, HL, LZ, GK, JC, and ZL are employees of Echo Biotech Co., Ltd. The other authors have no conflict of interest to declare.

Ethical Statement: The authors are accountable for all aspects of the work in ensuring that questions related to the accuracy or integrity of any part of the work are appropriately investigated and resolved. The study was officially approved by the ethics committee of the Beijing Friendship Hospital (approval ID: L-2018-030). All procedures performed in this study involving human participants were in accordance with the Declaration of Helsinki (as revised in 2013). Informed consent was taken from all the patients.

Open Access Statement: This is an Open Access article distributed in accordance with the Creative Commons Attribution-NonCommercial-NoDerivs 4.0 International License (CC BY-NC-ND 4.0), which permits the noncommercial replication and distribution of the article with the strict proviso that no changes or edits are made and the original work is properly cited (including links to both the formal publication through the relevant DOI and the license). See: https://creativecommons.org/licenses/by-nc-nd/4.0/.

\section{References}

1. Siegel RL, Miller KD, Jemal A. Cancer statistics, 2018. CA Cancer J Clin 2018;68:7-30.

2. Skolarus TA, Wolf AM, Erb NL, et al. American Cancer Society prostate cancer survivorship care guidelines. CA Cancer J Clin 2014;64:225-49.

3. Grasso CS, Wu YM, Robinson DR, et al. The mutational 
landscape of lethal castration-resistant prostate cancer. Nature 2012;487:239-43.

4. Zadra G, Ribeiro CF, Chetta P, et al. Inhibition of de novo lipogenesis targets androgen receptor signaling in castration-resistant prostate cancer. Proc Natl Acad Sci U S A 2019;116:631-40.

5. Li Z, Bishop AC, Alyamani M, et al. Conversion of abiraterone to D4A drives anti-tumour activity in prostate cancer. Nature 2015;523:347-51.

6. Li Z, Alyamani M, Li J, et al. Redirecting abiraterone metabolism to fine-tune prostate cancer anti-androgen therapy. Nature 2016;533:547-51.

7. de Bono JS, Logothetis CJ, Molina A, et al. Abiraterone and increased survival in metastatic prostate cancer. $\mathrm{N}$ Engl J Med 2011;364:1995-2005.

8. Ryan CJ, Smith MR, de Bono JS, et al. Abiraterone in metastatic prostate cancer without previous chemotherapy. N Engl J Med 2013;368:138-48.

9. Tsaur I, Becker C, Thelen P, et al. Splice variant AR-V7 : Is it time for its routine use as a predictive marker in prostate cancer? Urologe A 2017;56:1164-7.

10. Sharp A, Coleman I, Yuan W, et al. Androgen receptor splice variant-7 expression emerges with castration resistance in prostate cancer. J Clin Invest 2019;129:192-208.

11. Navarro HI, Goldstein AS. HoxB13 mediates AR-V7 activity in prostate cancer. Proc Natl Acad Sci U S A 2018;115:6528-9.

12. Markowski MC, Silberstein JL, Eshleman JR, et al. Clinical Utility of CLIA-Grade AR-V7 Testing in Patients With Metastatic Castration-Resistant Prostate Cancer. JCO Precis Oncol 2017.

13. Scher HI, Graf RP, Schreiber NA, et al. Nuclear-specific AR-V7 Protein Localization is Necessary to Guide Treatment Selection in Metastatic Castration-resistant Prostate Cancer. Eur Urol 2017;71:874-82.

14. Tommasi S, Pilato B, Carella C, et al. Standardization of CTC AR-V7 PCR assay and evaluation of its role in castration resistant prostate cancer progression. Prostate 2019;79:54-61.

15. Scher HI, Lu D, Schreiber NA, et al. Association of ARV7 on Circulating Tumor Cells as a Treatment-Specific

Cite this article as: Wang C, Liu X, Li H, Zhao L, Kong G, Chen J, Li Z, Qi J, Tian Y, Zhang F. Urinary exosomebased androgen receptor-variant 7 detection in metastatic castration-resistant prostate cancer patients. Transl Androl Urol 2022;11(2):202-212. doi: 10.21037/tau-21-1136
Biomarker With Outcomes and Survival in Castration-

Resistant Prostate Cancer. JAMA Oncol 2016;2:1441-9.

16. Ignatiadis M, Lee M, Jeffrey SS. Circulating Tumor Cells and Circulating Tumor DNA: Challenges and Opportunities on the Path to Clinical Utility. Clin Cancer Res 2015;21:4786-800.

17. Seitz AK, Thoene S, Bietenbeck A, et al. AR-V7 in Peripheral Whole Blood of Patients with Castrationresistant Prostate Cancer: Association with Treatmentspecific Outcome Under Abiraterone and Enzalutamide. Eur Urol 2017;72:828-34.

18. To SQ, Kwan EM, Fettke HC, et al. Expression of Androgen Receptor Splice Variant 7 or 9 in Whole Blood Does Not Predict Response to Androgen-Axis-targeting Agents in Metastatic Castration-resistant Prostate Cancer. Eur Urol 2018;73:818-21.

19. Tkach M, Théry C. Communication by Extracellular Vesicles: Where We Are and Where We Need to Go. Cell 2016;164:1226-32.

20. Houali K, Wang X, Shimizu Y, et al. A new diagnostic marker for secreted Epstein-Barr virus encoded LMP1 and BARF1 oncoproteins in the serum and saliva of patients with nasopharyngeal carcinoma. Clin Cancer Res 2007;13:4993-5000.

21. Castellanos-Rizaldos E, Grimm DG, Tadigotla V, et al. Exosome-Based Detection of EGFR T790M in Plasma from Non-Small Cell Lung Cancer Patients. Clin Cancer Res 2018;24:2944-50.

22. Kannan A, Wells RB, Sivakumar S, et al. Mitochondrial Reprogramming Regulates Breast Cancer Progression. Clin Cancer Res 2016;22:3348-60.

23. Del Re M, Biasco E, Crucitta S, et al. The Detection of Androgen Receptor Splice Variant 7 in Plasma-derived Exosomal RNA Strongly Predicts Resistance to Hormonal Therapy in Metastatic Prostate Cancer Patients. Eur Urol 2017;71:680-7.

24. Woo HK, Park J, Ku JY, et al. Urine-based liquid biopsy: non-invasive and sensitive AR-V7 detection in urinary EVs from patients with prostate cancer. Lab Chip 2018;19:87-97.

(English Language Editor: L. Huleatt) 